\title{
Including ELSI research questions in newborn screening pilot studies
}

\author{
Aaron J. Goldenberg, PhD., MPH ${ }^{1}$, Michele Lloyd-Puryear, MD, PhD ${ }^{2}$, Jeffrey P. Brosco, MD, PhD ${ }^{3}$, \\ Bradford Therrell, $\mathrm{PhD}^{4}$, Lynn Bush, $\mathrm{PhD}^{5}$, Susan Berry, $\mathrm{MD}^{6}$, Amy Brower, $\mathrm{PhD}^{8}$, \\ Natasha Bonhomme ${ }^{7}$, Bruce Bowdish, PhD $^{8}$, Denise Chrysler, JD ${ }^{9}$, Angus Clarke, DM, FRCP ${ }^{10}$, \\ Thomas Crawford, MD ${ }^{11}$, Edward Goldman, JD ${ }^{12}$, Sally Hiner ${ }^{13}$, R. Rodney Howell, MD, FAAP ${ }^{14}$, \\ David Orren, JD ${ }^{15}$, Benjamin S. Wilfond, $\mathrm{MD}^{16}$ and \\ Michael Watson, PhD, FACMG ${ }^{5}$ for the Bioethics and Legal Workgroup of the Newborn \\ Screening Translational Research Network
}

\begin{abstract}
Background: The evidence review processes for adding new conditions to state newborn screening (NBS) panels rely on data from pilot studies aimed at assessing the potential benefits and harms of screening. However, the consideration of ethical, legal, and social implications (ELSI) of screening within this research has been limited. This paper outlines important ELSI issues related to newborn screening policy and practices as a resource to help researchers integrate ELSI into NBS pilot studies.
\end{abstract}

Approach: Members of the Bioethics and Legal Workgroup for the Newborn Screening Translational Research Network facilitated a series of professional and public discussions aimed at engaging NBS stakeholders to identify important existing and emerging ELSI challenges accompanying NBS.

Results: Through these engagement activities, we identified a set of key ELSI questions related to (1) the types of results parents may receive through newborn screening and (2) the initiation and implementation of NBS for a condition within the NBS system.

Conclusion: Integrating ELSI questions into pilot studies will help NBS programs to better understand the potential impact of screening for a new condition on newborns and families, and make crucial policy decisions aimed at maximized benefits and mitigating the potential negative medical or social implications of screening.

Genetics in Medicine (2019) 21:525-533; https://doi.org/10.1038/s41436018-0101-x

Keywords: Newborn screening; Ethics; Pilot studies; Research; ELSI

\section{INTRODUCTION}

Newborn screening (NBS), a population-based screening program, is required for every newborn in the United States. ${ }^{1,2}$ For over 50 years, NBS has led to the identification and early treatment of tens of thousands of newborns for selected birth defects. ${ }^{3,4}$ US state-based NBS programs began with screening for phenylketonuria in the 1960s and expanded to include a variety of other conditions as screening technologies developed, clinical understanding improved, and treatments became available. Each state ultimately decides which conditions to include, or exclude, in their statewide NBS program. In addition, the Secretary of Health and Human Services, considering the advice of the Advisory Committee for Heritable Disorders of Newborns and Children (ACHDNC), recommends conditions for screening, termed the Recommended Uniform Screening Panel (RUSP). ACHDNC and state decisions to recommend the addition of new conditions include processes that examine the evidence

\footnotetext{
${ }^{1}$ Department of Bioethics, Center for Genetic Research Ethics and Law, Case Western Reserve University, Cleveland, Ohio, USA; ${ }^{2}$ Parent Project Muscular Dystrophy, Hackensack, New Jersey, USA; ${ }^{3}$ Institute for Bioethics and Health Policy, Mailman Center for Child Development, and Department of Pediatrics, University of Miami, Miami, Florida, USA; ${ }^{4}$ University of Texas Health Science Center at San Antonio and National Newborn Screening and Global Resource Center, Austin, Texas, USA; ${ }^{5}$ Pediatric Clinical Genetics and Program in Women \& Children's Bioethics, Columbia University Medical Center, New York, New York, USA; ${ }^{6}$ Departments of Pediatrics and Genetics, Cell Biology \& Development, Division of Genetics and Metabolism, University of Minnesota, Minneapolis, Minnesota, USA; ${ }^{7}$ Genetic Alliance, Washington, DC, USA; ${ }^{8}$ American College of Medical Genetics and Genomics, Bethesda, Maryland, USA; ${ }^{9}$ Network for Public Health Law - Mid-States Region, Ann Arbor, Michigan, USA; ${ }^{10}$ Department of Medical Genetics, University Hospital Wales, Cardiff, Wales, UK; ${ }^{11}$ Departments of Neurology and Pediatrics, Johns Hopkins University, Baltimore, Maryland, USA; ${ }^{12}$ University of Michigan Law School and member of the Center for Bioethics in Social Science and Medicine, The University of Michigan, Ann Arbor, Michigan, USA; ${ }^{13}$ Michigan Public Health Institute, Okemos, Michigan, USA; ${ }^{14}$ Miller School of Medicine, University of Miami, Miami, Florida, USA; ${ }^{15}$ Minnesota Department of Health, Minneapolis, Minnesota, USA; ${ }^{16}$ Department of Pediatrics, Treuman Katz Center for Pediatric Bioethics Seattle Children's Research Institute, University of Washington School of Medicine, Seattle, Washington, USA. Correspondence: for the Bioethics and Legal Workgroup of the Newborn Screening Translational Research Network Aaron J. Goldenberg (ajg10@case.edu)
} 
of the potential net benefit of screening, the ability of states to screen for the disorder, and the availability of effective treatments. ${ }^{5}$ Historically, the consideration of ethical, legal, and social implications (ELSI) within these evidence review processes has been limited, generally because researchers have not included a systematic study of ELSI issues within NBS research studies. The goal of this paper is to systematically outline important ELSI issues related to NBS policy and practices as a resource to help researchers integrate ELSI into NBS pilot studies, "systematic investigations or public health activities that are designed to evaluate the efficacy and safety of incorporating a new test or condition on a populationbased level into state NBS programs."

As researchers, clinicians, NBS policy makers, and federal agencies have reviewed evidence related to the harms and benefits of mandating screening of newborns for a new condition, some of their deliberations have included ELSI issues including discussions of parental permission, the availability of treatments and their costs, and the disparity caused by state-based variability in the number and types of conditions screened. Within the context of NBS, previous NBS ELSI research studies have examined issues such as the impact of false positive screens or uncertain results on families and the ethical implications of storing and using residual blood spots for further research. ${ }^{7-11}$ However, the extent of this research has been limited and except in a few cases, ${ }^{12,13}$ most ELSI studies associated with NBS have not been conducted as part of pilot studies investigating the benefits and harms of adding a new condition. This has led to a paucity of empirical ELSI data that could contribute to the evidence review process designed to evaluate new conditions for NBS. Any systematic evaluation of benefits or harms regarding expanding NBS panels is hindered by this research gap. Additionally, continued advances in our understanding of disease and the introduction of new screening technologies continue to raise ELSI concerns such as the disclosure of uncertain results, when to report possible carrier status, or the possible psychosocial impact from false positive screens. These concerns and others warrant further research to improve understanding of the issues and provide a basis for policy decisions. ${ }^{14-18}$

This paper systematically outlines the crucial ELSI issues arising in NBS to help researchers better integrate robust consideration of these issues into NBS pilot studies. To do so, we have identified nine key ELSI questions that represent important ethical or social challenges for NBS policy and practice. Our goal is to promote incorporation of these questions into NBS pilot studies to add to the evidence base for making better informed policy and practice decisions. We posit that ELSI data collected as part of pilot studies can allow the ACHDNC and state NBS programs to better address ELSI more systematically in their evidence review processes. This in turn will help maximize the benefits of screening and mitigate any potential negative impact on newborns, their families, and the public health system through informed policy and practice changes. We recognize that there are also a number of research ethics challenges related to the design and implementation of NBS pilot studies themselves, including human research subject protections issues, related recruitment, and study design. However, these important research process questions are beyond the scope of this paper because our focus is on the ELSI questions that impact the decision to add a condition to NBS programs. So, for example, questions related to the potential need for parental permission for new screened conditions are raised in this paper, but consent requirements for enrolling participants in a pilot study are not. Future work in this area will be needed to address these important, yet distinct, research ethics questions.

\section{DELIBERATIVE APPROACH}

The key questions presented in this paper were developed through a series of professional and public discussions aimed at engaging multiple NBS stakeholders to help identify important existing and emerging ELSI challenges accompanying NBS. These questions began with activities of a Parent Project Muscular Dystrophy (PPMD) ${ }^{19}$ workgroup focused on ELSI issues raised by the potential addition of Duchenne muscular dystrophy (DMD) to NBS panels. Members of the National Institutes of Health's (NIH) Eunice Kennedy Shriver National Institutes of Child Health and Human Development's (NICHD) Newborn Screening Translational Research Network's (NBSTRN). ${ }^{20}$ The Steering Committee and Bioethics and Legal Issues Workgroup then discussed these questions, developing a series of general ethical issues that should be addressed by any NBS pilot study. This workgroup is made up of more than 20 NBS stakeholders whose goal is to provide expert advice on issues pertaining to newborn screening research. Included are NBS program officials, lawyers, bioethicists and social scientists, geneticists, laboratory officials, lawyers, and biomedical researchers. An early draft of these key questions was presented and discussed at the NBSTRN's annual network meeting in Fall 2016. This meeting included over 100 NBS stakeholders including NBS policy makers, laboratory directors, NBS researchers, and representatives of disease advocacy organizations. Finally, these questions were posted and discussed on a public forum, the NBS Public Square hosted by Genetic Alliance through Baby's First Test, ${ }^{21}$ the National Newborn Screening Clearinghouse, and funded by Health Resources and Services Administration (HRSA). Each of the engagement exercises was meant to solicit informed opinions from NBS stakeholders to iteratively refine and enhance the content of this paper. For example, stakeholders from the NBSTRN Network Meeting aided significantly in refining our discussion on the potential harms of false positives and false negatives. Additionally, responses from our online discussion forum led to the inclusion of questions related to equity health disparities.

\section{ESSENTIAL ELSI RESEARCH QUESTIONS}

The following sections review nine key ELSI questions divided the into two main categories: (1) ELSI issues associated with the results of screening, and (2) ELSI issues anticipated in the initiation and implementation of NBS for a condition within the NBS system. Specific examples of possible research 
Table 1 Sample ELSI research questions Key ELSI questions

\section{Issues related to NBS results}

What are the potential ELSI of positive screening results related to a new condition?
Potential data sources

-Families

- Clinicians

-Administrative databases

\begin{abstract}
What are the potential ELSI implications of false positive • Families screening results related to a new condition?

- Clinicians

-Administrative databases
\end{abstract}

What are the potential ELSI of false negative screening results related to a new condition?

What are the potential ELSI of obtaining and reporting carrier status related to a new condition?

-Families

- Clinicians

-Administrative databases

What are the potential ELSI of indeterminate results related to a condition?
-Families

- Clinicians

-Administrative databases

\section{Issues related to the NBS system}

What are the cost or resource allocation implications for adding a new condition to the RUSP or a state panel?
- State NBS programs

-Public health departments, other state agencies - Clinicians, professional
Sample ELSI research questions

-Do caregivers treat an infant differently when a presymptomatic diagnosis is made?

-What are the potential harmful or beneficial effects of an NBS diagnosis on maternal-infant bonding or other family dynamics?

-Are there potential harms from subsequent diagnostic testing (which may be invasive) and treatment and how do these harms impact the net benefits of screening?

-What are the financial costs of diagnosis and follow-up? What is the system-wide cost?

-Do caregivers treat an infant differently as a result of receiving a false positive screen result?

-Are there long-lasting psychological consequences for a positive screening test in infants who do not have a condition? What is the effect of a false positive on maternal-infant bonding?

-Are there potential harms from subsequent diagnostic testing?

-What are the financial costs of diagnosis and follow-up? What is the system-wide cost?

-What is the preventable morbidity and mortality related to false negative screening results?

-Do normal NBS results provide false reassurance to parents (e.g., cause people to ignore symptoms of serious illness? or could cause a unnecessary diagnostic odyssey for families later in life?)

- How does knowledge of carrier status impact the newborn/families? What is the cost/ benefit to the newborn? To the family? Of disclosing carrier status? oDoes knowledge of carrier status improve understanding the risk of developing diseases?

-Does this knowledge lead to stigmatization, concerns about life expectancy, changes in lifestyle choices, or decisions about having more children? Does it affect other family members?

oWhat are the financial costs of follow-up? What is the system-wide cost?

-Does knowledge of potential illness provide families with reassurance that they will be able to intervene at the earliest possible moment? Does it lead to anxiety and concern about even minor symptoms?

-Are there potential harms from subsequent diagnostic testing and follow-up?

-What are the financial costs of diagnosis and follow-up? What is the system-wide cost?

-Are state NBS programs ready to implement the new screening test, or does it require radically new procedures, equipment, or expertise? What are the likely costs (including case follow-up)?

-What are the opportunity costs, if any, of expanding to 
Table 1 continued

\begin{tabular}{|c|c|c|}
\hline Key ELSI questions & Potential data sources & Sample ELSI research questions \\
\hline & $\begin{array}{l}\text { organizations, health care } \\
\text { organizations, general public }\end{array}$ & $\begin{array}{l}\text { include the new condition? } \\
\text {-Is there a sufficient number of clinicians trained to treat } \\
\text { the condition? What is their geographic distribution? } \\
\text {-What is the system-wide financial cost of diagnosis and } \\
\text { treatment? } \\
\text {-Are the prevalence and impact of the condition sufficient } \\
\text { to justify the cost? Are there plans for long-term follow-up } \\
\text { to judge impact of programs? }\end{array}$ \\
\hline $\begin{array}{l}\text { What are the health disparities or equity considerations } \\
\text { related to adding a new condition to the RUSP or a state } \\
\text { panel? }\end{array}$ & $\begin{array}{l}\text {-NBS programs, families, NBS } \\
\text { researchers, general public, } \\
\text { health care organizations }\end{array}$ & $\begin{array}{l}\text {-Do decisions about how to screen for a condition have } \\
\text { implications for which populations are most likely to be } \\
\text { diagnosed (e.g., CF screening)? } \\
\text {-Are population-level results of NBS likely to affect one } \\
\text { population in particular (e.g., reveal high rates of } \\
\text { infectious disease or stigmatizing condition)? } \\
\text {-What factors will influence access to confirmatory testing } \\
\text { and treatment (e.g., health insurance, geography, culture, } \\
\text { race/ethnicity)? }\end{array}$ \\
\hline $\begin{array}{l}\text { What are the potential implications for public/parental } \\
\text { trust in the NBS system or health department that might } \\
\text { arise because of adding a new condition? }\end{array}$ & $\begin{array}{l}\text {-NBS programs, families, } \\
\text { clinicians, general public, health } \\
\text { care organizations }\end{array}$ & $\begin{array}{l}\text {-Do false negative/false positives weaken faith in NBS } \\
\text { programs and the ability of health departments to provide } \\
\text { accurate and helpful information? } \\
\text { •Is there transparency in the process of adding a new } \\
\text { condition to a panel, the implementation of screening } \\
\text { tests, and approach to follow-up and treatment? }\end{array}$ \\
\hline $\begin{array}{l}\text { Does a condition raise any concerns regarding parental } \\
\text { permission or challenges to the ethical or social } \\
\text { justification for requiring population-based screening? }\end{array}$ & $\begin{array}{l}\text {-NBS programs, families, } \\
\text { clinicians, general public }\end{array}$ & $\begin{array}{l}\text {-Does the condition have such a high benefit:cost ratio } \\
\text { that the general public and nearly all families would agree } \\
\text { that NBS should be universal? Or would many reasonable } \\
\text { people choose to opt out (e.g., later-onset condition with } \\
\text { ambiguous benefits of treatment)? }\end{array}$ \\
\hline
\end{tabular}

NBS newborn screening, RUSP Recommended Uniform Screening Panel, CF cystic fibrosis, ELS/ ethical, legal, and social implications

questions are provided in Table 1. Each of these sets of questions may impact one or more NBS stakeholder group, including (1) newborns, (2) families, (3) state NBS programs, (4) clinicians and the larger health care delivery system, and (5) the general population. We also recognize that ELSI issues vary depending on the specific condition, and some may raise unique challenges for families and other NBS stakeholders. Additionally, not all pilot studies will need, or be able, to answer every question from each of these sets of ELSI issues.

\section{ELSI questions raised by NBS results related to screening for a condition}

The results-related ELSI questions focus on outcomes related to the analytical and clinical validity or utility of the screening test or of the subsequent diagnostic tests. From any screen there are a number of possible results including screen positive (out-of-range screens), screen-negative, or false positive/false negative screen. NBS programs continually work to minimize the potential ambiguity or negative implications of any of these screening results. For example, programs strive to reduce the number of false positive and false negative screening results to mitigate potential medical or psychosocial harm to newborns and families. Nevertheless, as NBS programs add conditions and utilize new technologies, there will be a need for ongoing ELSI research to continually assess the potential impacts, both benefits and harms, that NBS results may have for families. In turn, these data can be utilized to make crucial policy decisions aimed at mitigating the potential negative medical or social implications of screening. Results of ELSI studies may, for example, lead to improved screening algorithms and inform decisions about the kind of results and way in which results should be reported. This includes understanding the impact of how the communication of screening results from NBS programs and health care providers may impact the screened infants and their families, including ensuring that accurate and useful information from the screening and diagnostic processes becomes a meaningful part of the child's health care record.

\section{Key question 1: what are the potential ELSI of positive screening results related to a condition?}

Screening tests do not provide a diagnosis, but rather provide an indication of increased risk. A diagnostic test establishes the presence (or absence) of a disorder. When the screening 
test result is positive or out of range and the diagnosis is confirmed, the family typically receives a diagnosis before the infant's disease is clinically observable-this is one of the primary goals of NBS.

ELSI. The ethical or social issues associated with a positive or out-of-range result relate primarily to assessing the benefits of screening and any potential medical harms and financial burdens associated with the diagnostic process following the screen. These concerns are especially salient for conditions where diagnostic testing and treatments are more invasive or where long-term medical impact or benefits of interventions are less clear. Additionally, as programs have expanded, there are increasing numbers of conditions with later-onset variants, further complicating diagnostic and treatment decisions. ${ }^{22}$ While these issues are a core concern for NBS programs generally, they raise a number of important ethical concerns related to the harms and benefits of screening. Additionally, an out-ofrange result may bring worry, confusion, anger, depression, and even despair to a family. ${ }^{23-25}$ However, to date there are few published studies that look at the potential adverse psychosocial or emotional impacts of health screening results for most individuals and families. ${ }^{26}$ Pilot studies that include an ELSI focus are needed to evaluate screening protocols for new conditions and compare the net benefits of screening with the potential harms of screening and diagnostic procedures and help to address this gap in the context of NBS. These studies could also address the impact of different approaches to education and communication aimed at helping families understand screening information, and identify those at highest risk for distress who may need further psychological support.

Key question 2: what are the potential ELSI of false positive screening results related to a new condition?

Some screening tests, unlike diagnostic tests, generate relatively large numbers of patients needing further evaluation to clarify initial screening results. The need for such reassessment may have implications for many stakeholders, particularly because the recall rate can vary significantly from state to state, depending on the screening protocol. While the goal is to minimize the number of patients recalled who do not have the screened condition, some patients, with further testing, will be shown to not have the disease and will therefore be considered to have a false positive screen.

ELSI. Too many false positive screens potentially weakens public confidence in NBS programs and diminishes the ability of health departments to provide a useful service to families. ${ }^{27}$ Other ELSI issues include concerns about the possible impact of false positive screening results on parental stress; bonding between parent and newborn; and cost, inconvenience, and potential invasiveness of additional medical procedures needed to establish a diagnosis. ${ }^{28}$ Previous research suggests limited psychosocial implications for most individuals and families, and studies have not shown increased health care utilization by parents with newborns who receive false positive screening results. ${ }^{29,30}$ However, the existing empirical research on false positive screens has been limited in diversity of populations included, lack of subgroup analyses, and the need for more generalizable quantitative data. Further research is needed to assess whether specific subgroups within the screened population may be at increased risk for higher levels of stress or other familial impacts of false positive screening results. Excessive numbers of false positive screening results may also put demands on state NBS program resources and the capacity of the health care system to respond. Studies of the financial costs of false positive screens could provide estimates of system-wide impact.

\section{Key question 3: what are the potential ELSI of false negative screening results related to a new condition?}

When implementing screening technology for a new condition, NBS programs must attempt to eliminate late diagnosed cases as a result of a false negative screening result while not overburdening the system with excessive recalls for further testing. This is particularly troublesome because a screennegative result is often interpreted as if a diagnosis of normalcy instead of a reduced risk of the presence of the condition. Thus, when screening procedures for a new condition reach the level of evidence necessary to justify using it in a state NBS program, the potential for false negative screening results should be very low, maximizing test sensitivity. The potential impact of a false negative result will greatly depend on the condition screened and the implications of postsymptomatic diagnosis.

ELSI. The primary ELSI of false negative results relate to the potential false reassurance that these results can cause for health care providers and parents, and the potential harm that can result from delayed diagnoses. Subsequently, like false positive screens, increased numbers of false negative screens may erode public trust and support for NBS programs. Including ELSI issues in pilot studies should help in understanding the impact of false negative findings relative to public trust. A key outcome of pilot studies aimed at assessing the potential for false negative screens could focus on creating policies and educational materials to help increase awareness about the potential for false negative screening results and to mitigate the potential harms to newborns and families if conditions are detected and diagnosed late.

\section{Key question 4: what are the potential ELSI of obtaining and reporting carrier status related to a new condition?}

Many of the conditions that may be piloted in NBS programs are inherited in a recessive manner, where both the mother and father are carriers of the condition. Thus, screening for these conditions may identify newborns who are also carriers of a condition and who may not be clinically affected. As a result, unique ELSI questions arise when a newborn's carrier 
status may be identified as part of the NBS process. As a practical matter, NBS already detects possible carrier status for many of the screened conditions including sickle cell disease, cystic fibrosis, and others. ${ }^{15}$ The precise laboratory techniques employed will determine the scale of this issue, i.e., what proportion of carriers would be identified through screening.

ELSI. The core ELSI issues center on debates regarding disclosure and potential benefits and harms of carrier status information for newborns and families, and their implications for state policy decisions to disclose, or not disclose, carrier results. $^{31}$ Being a carrier for conditions screened may have health implications for individuals either in childhood or as an adult, including when they eventually make their own reproductive choices. Additionally, there are arguments for assessing the social implications of screening for family benefit beyond that of the newborn. For example, carrier results may be important for parents considering their own future reproductive choices. ${ }^{30}$ NBS laboratories generally report carrier findings as part of their perceived ethical or legal obligation to disclose all testing results. Similarly, parents may also lose trust in the NBS system if they perceive that the programs are withholding information such as carrier status from them.

Alternatively, arguments could be made that there is less of an ethical obligation to disclose carrier status, or even an obligation to not disclose, if the information would have no immediate impact on the health of a newborn or child, ${ }^{32}$ and may be misinterpreted by some as having the disease. This debate raises important questions about how we assess the social implications of screening and the impact of NBS information on newborns and families.

Pilot studies could give researchers and states the opportunity to collect data on the impact of reporting carrier status to families should the methodology under study have the potential for such revelations. These data could be used to develop more informed policies regarding decisions to report carrier status results to health care providers and their handling of this information (i.e., whether and when to provide to parents). For example, studies could investigate parental perceptions on how carrier information identified as part of NBS may be of significant value for the child across their lifespan and whether the information is appropriately understood. Therefore, aside from programmatic decisions about reporting protocols, it is important to consider the ELSI issues for individuals and their families who might receive possible carrier status results, and how that information may be of value to their health or reproductive choices. $^{33}$

\section{Key question 5: what are the potential ELSI of} indeterminate results related to a condition?

In NBS there are sets of results for which the implications of the information obtained through screening may be unclear. This may be especially true as more complex conditions are added to screening panels. The addition of conditions with an unknown age of onset, variable phenotypic features, unknown penetrance, or unclear severity creates a situation in which parents might have uncertain knowledge concerning the presence or absence of disease, even after diagnostic testing is conducted. $^{34}$

ELSI. When indeterminate results are returned to parents, there may be increased anxiety and stress related to when, or if, symptoms will occur in their children or themselves (for example, as with Krabbe disease or cystic fibrosis transmembrane conductance regulator-related metabolic syndrome). ${ }^{35}$ Delayed onset of a condition has the potential for labeling newborns as "diseased" before any actual manifestation of the disease, and without knowledge of potential morbidity. Such newborns may become "patients-in-waiting" as families and affected individuals embark on an uncertain future or lengthy diagnostic odyssey. ${ }^{9}$ Pilot studies of conditions with variable penetrance or variable phenotypic features can document the potential emotional and financial costs before such conditions are considered for inclusion in the RUSP. For example, a pilot study could assess the potential impact that receiving indeterminate or unclear results may have on families, including reassurance that they would be able to intervene at the earliest possible moment if symptoms arise, or exploring whether these results ultimately increase anxiety with regard to an unknown future and raise concerns about even minor symptoms.

\section{ELSI questions related to the NBS system}

NBS system ELSI issues focus on the impact of initiation and implementation of a new condition on a state NBS program or a state's public health or health care delivery systems. These questions begin with the decision to consider screening for a particular condition and represent the implications that adding a condition (and its associated screening and diagnostic tests) could have on the state health department (medical foods, child health insurance programs, etc.), the NBS program, screening laboratory, or health care delivery systems.

\section{Key question 6: what are the cost or resource allocation implications for adding a new condition to the RUSP or a state panel?}

NBS programs require a great deal of resources to run an effective screening program for all infants born within their state. Costs to state programs are not merely associated with the screening process itself, but also cover a number of crucial components of the entire NBS system including parent education, laboratory needs, and follow-up services. ${ }^{36}$ If the addition of a condition to the program's NBS panel would be costly to the state NBS programs (equipment, training, personnel, and other resources), and implementation might impact the program's ability to function effectively in other areas, then assessing the net harms and benefits of screening 
for a particular condition is crucial to the decision-making process.

ELSI. If adding a new condition to an NBS panel disrupts a state's ability to maintain an effective NBS program, then expansion may prevent a program from fulfilling its social and ethical obligation to ensure that all newborns born within a state have the opportunity to be screened. Therefore, program costs/resources related to new condition implementation should be an integral part of the piloting process. These costs evaluations should include assessing the cost of educating parents, policy makers, health professionals, and laboratory specialists; building or incorporating results reporting infrastructure, and supporting public relations activities, including development of related materials (pamphlets, videos, etc.). For some resource-limited programs, addition of new conditions for NBS may be more difficult than for resource-rich programs, and additional support may be necessary.

\section{Key question 7: what are the health disparities or equity considerations related to adding a new condition to the RUSP or a state panel?}

Distributive justice questions in NBS include the availability and accessibility of short-term and long-term follow-up services and treatments. While an NBS program may have a variety of resources available for newborns and their families, there may be challenges for medically underserved and/or geographically isolated families to access condition-related health care services at either the state or local levels.

ELSI. Like resource allocation, equity considerations in NBS center on the ethical duty to assure that all newborns in a state have the opportunity to be screened and have appropriate follow-up services. ${ }^{2}$ Barriers to services may include a lack of access to medical foods and formulas not covered by insurers, ${ }^{37}$ expensive drugs, and difficulty obtaining expensive diagnostic procedures or medical interventions associated with a condition. All have the potential to increase inequities for families and populations. In some locations, sufficient subspecialty physician services may not exist and regional resources may be required. Pilots should include an assessment of how NBS programs and the larger health care system could mitigate medical and related service inequities using approaches like telemedicine and regional service networks.

\section{Key question 8: what are the potential implications for public/parental trust in the NBS system or health department that might arise because of adding a new condition?}

Knowledge about NBS and its requirement generally is low among parents and families. ${ }^{38}$ This awareness is exacerbated by the poor communication practices between NBS programs and health care practitioners. Therefore, improving awareness and education about the NBS system is a key area of concern for state programs as patients become more active in their own health care and especially, as programs grow to include more conditions. Trust in the NBS system is crucial to the success of screening and follow-up services.

ELSI. When developing pilots to study the implementation of a new condition, it is important to assess the potential impact that expanding a state NBS panel may have on public trust and other public perceptions. ${ }^{39,40}$ Such assessments may require examining perceptions about program transparency and the decision-making process for adding new conditions, including how best to promote and manage input from parents, advocacy organizations, and other stakeholders. Considerations should include the potential for public engagement and education concerning the addition of a new condition. It is vital to assess whether unique characteristics of a new condition will merit special considerations when returning screening results to individuals who are members of populations already exhibiting mistrust of the government or the health care system. For example, if a subgroup of the population has a higher risk of false positive screens, targeted educational and engagement activities may be required to that build greater trust in the NBS system as part of the larger health care community. In addition, due to the complexity of NBS technologies, treatments, and disorders, communicating the purpose of, and results for, a pilot must include language and dialogue understandable to health care providers and families.

\section{Key question 9: does a condition raise any concerns regarding parental permission or challenges to the ethical or social justification for requiring population-based screening?}

NBS is a public health requirement, and in the United States parents are not required to give their consent. Justification for the screening requirement is predicated on the principle that requiring active parental permission about NBS is not necessary because the benefits outweigh the potential harm (s) of screening. Most state programs (although not all) allow parents to opt out of screening for religious or other specified reasons. ${ }^{41}$ As the complexity of genetic diseases is better understood as a result of population screening, some may argue that parents should be asked to opt in for NBS that may include conditions for which clinical symptoms arise later in life and not during childhood. That is, unless conditions need emergent diagnosis and treatment, there is less justification for a requirement to have NBS. For example, an abnormal result for some lysosomal storage disorders (LSDs) might mean that the baby is an asymptomatic carrier, or that he (she) will experience late onset of the disease with milder symptoms, or that he (she) needs treatment immediately to prevent serious outcomes, or other possibilities, depending on the LSD. Insurance companies may not pay for the additional testing required for a definitive diagnosis. 
ELSI. There are a number of different approaches programs can take with regard to parental permission, including a variety of opt-out and opt-in options. ${ }^{34}$ ELSI issues surrounding parental permission center on the need to weigh the public health necessity and benefits of screening with parental choice. These ELSI questions are compounded when interventions for diagnosed infants are not medical treatments. As an example, early interventions may be initiated to minimize a cognitive delay in a condition such as fragile $\mathrm{X}$ rather than working to eliminate the underlying defect in cognition. ${ }^{11}$ Obtaining parental permission for some NBS conditions while not requiring permission for others (thereby combining optin and opt-out processes) could create a tiered screening approach. ${ }^{42}$ Thus, the benefits of universal screening for conditions where the need for early detection and the availability of effective interventions are more certain would exist while allowing parents to choose screening for conditions that are in pilot study or do not meet accepted criteria required for universal mandate. The potential harms for a two-tiered approach requires closer examination particularly with respect to the potential for creating an overly complicated system that might lead to increased processing errors or decreased patient participation and social inequities. Pilot studies could include questions and methodological designs that could test different types of parental permission and help programs assess the impact of different approaches.

\section{APPLYING KEY ELSI QUESTIONS IN PILOT STUDIES}

These questions are intended to help research teams systematically consider the kinds of empirical ethics questions that can be assessed within pilot studies. However, the utilization of these questions within the development of pilot studies will ultimately be shaped by the condition being studied. It is our hope that research teams will develop a set of conditionspecific ELSI questions to guide their own inquiry into the benefits and harms of population screening for their proposed condition. The purpose of Table $\mathbf{1}$ is to provide teams of clinicians, advocates, and investigators with sample empirical questions that could aid in identifying and assessing ELSI issues related to a specific condition.

In addition to the use of this tool, we also encourage researchers designing pilot studies to include researchers engaged in ELSI-related work in the planning and implementation of NBS pilots. Doing so will allow for more robust identification of ELSI challenges related to a condition, or sets of conditions, and will aid in the integration of ELSI research methodologies into pilots, including surveys, interviews, and other quantitative and qualitative research methods.

We recognize that the challenges facing NBS pilots to integrate ELSI questions go beyond the availability of ethics expertise. Funding agencies do not usually include specific ELSI concerns in assessing pilot study designs for NBS research projects. Researchers often lack sufficient funding or resources to include ELSI concerns in their projects. Relatively few ELSI scholars are trained in empirical research methods that can be easily linked to pilot studies. Federal and state NBS policy groups do not require the same level of evidence for ELSI questions as they do for screening tests, diagnostic evaluation, and clinical outcomes. Engaging NBS stakeholders to create innovative approaches to NBS research is necessary to integrate ELSI questions into NBS pilot studies.

\section{CONCLUSION}

NBS pilot studies provide an invaluable opportunity to explicitly address the ELSI issues of screening by assessing the potential benefits and harms of screening and follow-up, including diagnostic procedures, and medical interventions/ treatments for newborns and their families. We have delineated important questions for researchers to help in determining which ELSI should be included in pilot studies with the goal of identifying research gaps; increasing the evidence base for assessing net benefits and harms of population-based screening for newborn conditions; and promoting enhanced collaboration between researchers, state NBS programs, clinicians, advocacy organizations, ethicists, and social scientists to develop new approaches to assessing these difficult questions.

It is also crucial to acknowledge that NBS is constantly evolving. For example, some conditions being considered for NBS may have interventions that fall outside "traditional medical treatments." While screening for these conditions may provide net benefit to newborns and families, they may challenge the traditional metrics that have been used to determine when a condition is appropriate for addition to NBS panels. Additionally, adding new testing technologies, such as genomic sequencing as an alternative or adjunct screening modality, also provides NBS programs with an opportunity to improve the speed and quality of screening. It will be essential that as screening programs evolve, the ELSI questions asked in pilot studies also reflect the changing landscape of NBS.

\section{ACKNOWLEDGEMENTS}

We would like to thank Joanne Adelberg for her support on this manuscript. The work presented in this manuscript funded in part by the Newborn Screening Translational Research Network (funded by the National Institutes of Health, Eunice Kennedy Shriver National Institute of Child Health \& Human Development -275201300011 C-6-0-1).

\section{DISCLOSURE}

The authors declare no conflict of interest.

\section{REFERENCES}

1. Watson MS, Marie YM,Lloyd-Puryear MA, et al. Newborn screening: toward a uniform screening panel and system-executive summary. Pediatrics. 2006;117(suppl 3):S296-\$307.

2. Grosse SD, Coleen $A B$, Kenneson $A$, et al. From public health emergency to public health service: the implications of evolving criteria for newborn screening panels. Pediatrics. 2006;117:923-929. 
3. Therrell BL Jr., Lloyd-Puryear MA, Camp KM, et al. Mann Inborn errors of metabolism identified via newborn screening: ten-year incidence data and costs of nutritional interventions for research agenda planning. Mol Genet Metab. 2014;113:14-26.

4. Kuehn BM. After 50 years, newborn screening continues to yield public health gains. JAMA. 2013;309.12:1215-1217.

5. Kemper AR. Newborn Screening Translationl Research Network, Annual Network Meeting, Washington, DC, USA. Accessed on September 8, 2016.

6. Advisory Committee on Heritable Disorders in Newborns and Children by Jeffrey Botkin, May 2106. In Rockville, MD.

7. Ross LF. Newborn screening for lysosomal storage diseases: an ethical and policy analysis. J Inherit Metab Dis. 2012;35:627-634.

8. Gurian EA, Daniel DK, Henry JJ, et al. Expanded newborn screening for biochemical disorders: the effect of a false-positive result. Pediatrics. 2006;117:1915-1921.

9. Botkin JR, Rothwell E, Anderson R, et al. Public attitudes regarding the use of residual newborn screening specimens for research. Pediatrics. 2012;129:231-238.

10. Timmermans $S$, Buchbinder $M$. Patients-in-waiting: living between sickness and health in the genomics era. J Health Soc Behav. 2010;51:408-423.

11. Hewlett J, Waisbren SE. A review of the psychosocial effects of falsepositive results on parents and current communication practices in newborn screening. J Inherit Metab Dis. 2006;29:677-682.

12. Goldenberg AJ, Comeau AM, Grosse SD, et al. Evaluating harms in the assessment of net benefit: a framework for newborn screening condition review. Matern Child Health J. 2016:20:693-700.

13. Wilfond BS, Richard BP, Fost N. Balancing benefits and risks for cystic fibrosis newborn screening: implications for policy decisions. J Pediatr. 2005;147:S109-S113.

14. Bailey DB, et al. Ethical, legal, and social concerns about expanded newborn screening: fragile $X$ syndrome as a prototype for emerging issues. Pediatrics. 2008;121:e693-e704.

15. Tarini BA, Aaron JG. Ethical issues with newborn screening in the genomics era. Annu Rev Genomics Hum Genet. 2012;13:381-393.

16. Goldenberg AJ, Richard RS. The ethical hazards and programmatic challenges of genomic newborn screening. JAMA. 2012;307.5:461-462.

17. Peake RW, Bodamer OA. Newborn screening for lysosomal storage disorders. J Pediatr Genet. 2017;6:51-60.

18. Rothenberg $\mathrm{KH}$, Bush LW. Reframing an "open future": the shifting landscape from NBS to NBSeq. The drama of DNA: narrative genomics. New York: Oxford University Press; 2014; p. 47-56.

19. 2018 Parent Project Muscular Dystrophy 401 Hackensack Avenue, 9th Floor, Hackensack, NJ 07601. Accessed on 10 February 2017.

20. The Newborn Screening Translational Research Network, American College of Medical Genetics and Genomics. Bethesda, MD Accessed www.nbstrn.org on 10/04/2017.

21. Public Square Baby's First Test | Newborn Screening | The Genetic Alliance, Washington, DC USA, Accessed on 10 April 2017.

22. Orsini JJ, Caggana M. Newborn screening for Krabbe disease and other lysosomal storage disorders: broad lessons learned. Int J Neonatal Screen. 2017:3:3.
23. Collins RE, Lopez LM, Marteau TM. Emotional impact of screening: a systematic review and meta-analysis. BMC Public Health. 2011;11:603

24. Tluczek A, Murphy K, Orland LC. Psychosocial consequences of falsepositive newborn screens for cystic fibrosis. Qual Health Res. 2010;21:174-186

25. Morrison DR, Clayton. EW. False positive newborn screening results are not always benign. Public Health Genomics. 2011;14.3:173.

26. Collins RE, Laureen ML, Theresa MM. Emotional impact of screening: a systematic review and meta-analysis. BMC Public Health. 2011;11:603.

27. Tarini BA. The current revolution in newborn screening: new technology, old controversies. Arch Pediatr Adolesc Med. 2007;161:767-772.

28. Kwon C, Philip MF. The magnitude and challenge of false-positive newborn screening test results. Arch Pediatr Adolesc Med. 2000; 154.7:714-718

29. Hewlett J, Waisbren SE. A review of the psychosocial effects of falsepositive results on parents and current communication practices in newborn screening. J Inherit Metab Dis. 2006;29:677-682.

30. Tarini BA, Clark SJ, Pilli $S$, et al. False-positive newborn screening result and future health care use in a state Medicaid cohort. Pediatrics. 2011;128:715-722.

31. Miller FA, Robert JS, Hayeems. RZ. Questioning the consensus: managing carrier status results generated by newborn screening. Am J Public Health. 2009:99:210-215.

32. Committee on Bioethics. Ethical and policy issues in genetic testing and screening of children. Pediatrics. 2013;131:620-622.

33. Hantash FM, Goos DG, Tsao D, et al. Qualitative assessment of FMR1 (CGG) $n$ triplet repeat status in normal, intermediate, premutation, full mutation, and mosaic carriers in both sexes: implications for fragile $X$ syndrome carrier and newborn screening. Genet Med. 2010; 12.3:162-173.

34. Chien YH, Lee NC, Huang HJ, et al. Later-onset Pompe disease: early detection and early treatment initiation enabled by newborn screening. $J$ Pediatr. 2011;158.6:1023-1027.

35. Levy $\mathrm{H}$, Farrell PM. New challenges in the diagnosis and management of cystic fibrosis. The Journal of pediatrics. 2015;166:1337-41.

36. Baily MA, Thomas HM. Ethics, evidence, and cost in newborn screening Hastings Cent Report. 2008;38:23-31.

37. Berry SA, Kenney MK, Harris KB, et al. Insurance coverage of medical foods for treatment of inherited metabolic disorders. Genet Med. 2013;15:978.

38. Campbell $E$, Ross LF. Parental attitudes regarding newborn screening of PKU and DMD. Am J Med Genet A. 2003;120:209-214.

39. Kathleen A, Zuckerman B, Sharfstein JM, et al. A public health response to emerging technology: expansion of the Massachusetts newborn screening program. Public Health Rep. 2001;116:122-131.

40. Botkin JR, Clayton EW, Fost NC, et al. Newborn screening technology: proceed with caution. Pediatrics. 2006;117:1793-1799.

41. Botkin JR, Rothwell E, Anderson RA, et al. Prenatal education of parents about newborn screening and residual dried blood spots: a randomized clinical trial. JAMA Pediatr. 2016:170:543-549.

42. Ross LF. Mandatory versus voluntary consent for newborn screening? Kennedy Inst Ethics J. 2010:20:299-328. 